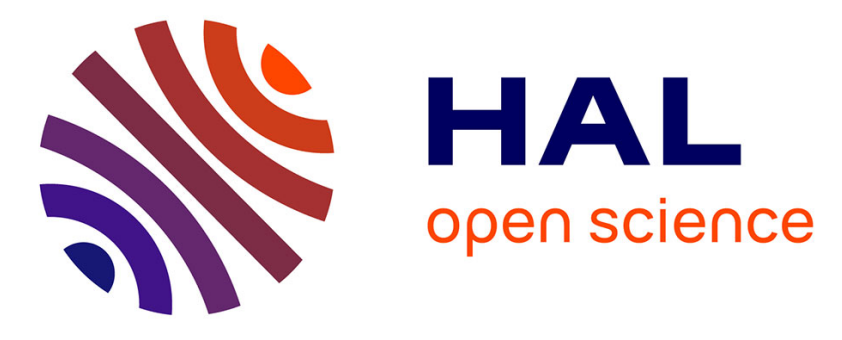

\title{
Evidence for the Existence of Elaborate Enzyme Complexes in the Paleoarchean Era
}

\author{
Bernd Reisinger, Josef Sperl, Alexandra Holinski, Veronika Schmid, Chitra \\ Rajendran, Linn Carstensen, Sandra Schlee, Samuel Blanquart, Rainer Merkl, \\ Reinhard Sterner
}

\section{To cite this version:}

Bernd Reisinger, Josef Sperl, Alexandra Holinski, Veronika Schmid, Chitra Rajendran, et al.. Evidence for the Existence of Elaborate Enzyme Complexes in the Paleoarchean Era. Journal of the American Chemical Society, 2014, pp.122-9. 10.1021/ja4115677 . hal-00924047

\section{HAL Id: hal-00924047 \\ https://inria.hal.science/hal-00924047}

Submitted on 1 Dec 2014

HAL is a multi-disciplinary open access archive for the deposit and dissemination of scientific research documents, whether they are published or not. The documents may come from teaching and research institutions in France or abroad, or from public or private research centers.
L'archive ouverte pluridisciplinaire HAL, est destinée au dépôt et à la diffusion de documents scientifiques de niveau recherche, publiés ou non, émanant des établissements d'enseignement et de recherche français ou étrangers, des laboratoires publics ou privés. 


\title{
Evidence for the existence of elaborate enzyme complexes in the Paleoarchean era
}

\author{
Bernd Reisinger ${ }^{1}$, Josef Sperl ${ }^{1}$, Alexandra Holinski ${ }^{1}$, Veronika Schmid ${ }^{1}$, Chitra Rajendran ${ }^{1}$, Linn \\ Carstensen', Sandra Schlee ${ }^{1}$, Samuel Blanquart ${ }^{2}$, Rainer Merkl ${ }^{*, 1}$, Reinhard Sterner ${ }^{*, 1}$ \\ ${ }^{1}$ Institute of Biophysics and Physical Biochemistry, University of Regensburg, Universitätsstraße 31, D-93053 Re- \\ gensburg, Germany \\ ${ }^{2}$ Equipe Bonsai, Institut National de Recherche en Informatique et en Automatique, INRIA Lille Nord Europe, 40 \\ avenue Halley, 59650 Villeneuve d'Ascq, France
}

Supporting Information Placeholder

\begin{abstract}
Due to the lack of macromolecular fossils, the enzymatic repertoire of extinct species has remained largely unknown to date. In an attempt to solve this problem, we have characterized a cyclase subunit (HisF) of the imidazole glycerol phosphate synthase (ImGP-S), which was reconstructed from the era of the last universal common ancestor of cellular organisms (LUCA). As observed for contemporary HisF proteins, the crystal structure of LUCA-HisF adopts the $(\beta \alpha)_{8}$-barrel architecture, one of the most ancient folds. Moreover, LUCA-HisF i) resembles extant HisF proteins with regard to internal two-fold symmetry, active site residues, and a stabilizing salt bridge cluster, ii) is thermostable and shows a folding mechanism similar to that of contemporary $(\beta \alpha)_{8}$-barrel enzymes, iii) displays high and specific catalytic activity, and iv) forms a stable and functional complex with the glutaminase subunit (HisH) of an extant ImGP-S. Furthermore, we show that LUCA-HisF binds to a reconstructed LUCA-HisH protein with high affinity. Our findings suggest that the evolution of highly specific enzymes and enzyme complexes has already been completed in the LUCA era, which means that sophisticated catalytic concepts such as substrate tunneling and allosteric communication existed already 4 billion years ago.
\end{abstract}

\section{Introduction}

Modern enzyme complexes are elaborate molecular machineries that have been optimized in the course of evolution for the efficient and specific processing of their substrates. One prominent example is the imidazole glycerol phosphate synthase (ImGP-S), a bi-enzyme complex which belongs to the family of glutamine amidotransferases ${ }^{1}$ and constitutes a branch-point connecting amino acid and nucleotide biosynthesis. ImGP-S consists of the cyclase subunit HisF and the glutaminase subunit HisH. HisF binds the substrate PRFAR and performs a cycloligase/lyase reaction that generates ImGP and AICAR, which are further used in histidine and de novo purine biosynthesis, respectively ${ }^{2}$ (Figure 1 ). The ammonia molecule required for this transformation is produced by the glutaminase subunit $\mathrm{HisH}$ and transported to the active site of HisF through an extended molecular channel. This channeling hampers diffusion of ammonia into bulk solvent and thus presumably prevents its protonation to the non-productive ammonium ion. Another special feature of the HisF/HisH complex is the tight coordination of the two enzymatic activities: Binding of PRFAR (or its analogue $N^{\prime}-$ [(5'-phosphoribosyl) formimino] -5-aminoimidazole-4carboxamide-ribonucleotide; ProFAR) to HisF results in an allosteric signal that leads to a several hundred-fold stimulation of the glutaminase activity of $\mathrm{HisH}^{3-5}$. This property precludes the hydrolysis of glutamine by HisH in the absence of an acceptor substrate at the active site of $\mathrm{HisF}^{6}$.

We were interested to find out whether the characteristics of modern HisF enzymes were already present in those species that colonized earth in a very early phase of biological evolution. A straightforward answer to this question is difficult due to the lack of macromolecular fossils. However, computational techniques of amino acid sequence reconstruction ${ }^{7,8}$ make it possible to travel back in time and to study extinct proteins ${ }^{9-16}$. In extreme cases, these algorithms enable us to study enzymes from the last universal common ancestor of cellular organisms (LUCA), which preceded the diversification of life and existed in the Paleoarchean era, $i$. e. at least 3.8 billion years (Gyr) and presumably 4.5 Gyr ago ${ }^{17}$.

Along these lines, we have previously computationally reconstructed the amino acid sequence of HisF from the LUCA era (LUCA-HisF) ${ }^{18}$. To this end, a set of 87 extant HisF and HisH proteins from the seven phylogenetic clades Crenarchaeota, Actinobacteria, Chlorobi, Cyanobacteria, Firmicutes, Proteobacteria, and Thermotogae has been used to determine a phylogenetic tree $t_{\text {HisF_HisH }}$ based on the CAT model ${ }^{19}$ (Figure 1). After having rooted this tree between the superkingdoms Archaea and Bacteria, we reconstructed a predecessor of HisF from Bacteria and Crenarchaeota as described ${ }^{19,20}$. Thus, although the precise lineage of the three 


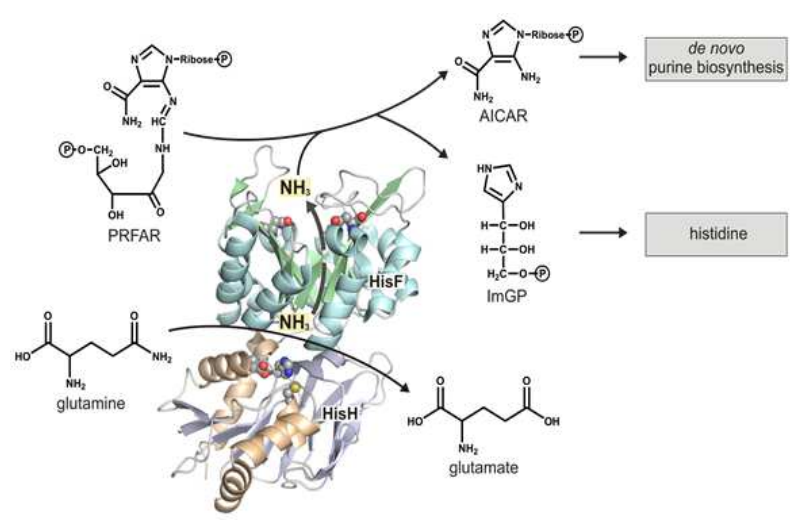

Figure 1. Reaction catalyzed by the heterodimeric ImGP synthase complex. The synthase subunit HisF catalyzes the reaction of $\mathrm{N}^{\prime}$-[(5'phosphoribulosyl)formimino]-5-aminoimidazole-4-carboxamide-ribonucleotide (PRFAR) with ammonia $\left(\mathrm{NH}_{3}\right)$ to imidazole glycerol phosphate (ImGP) and 5 -aminoimidazole-4-carboxamide ribotide (AICAR). ImGP is further utilized in the synthesis of histidine, whereas AICAR is an intermediate in de novo purine biosynthesis, rendering HisF a branch-point enzyme of amino acid and nucleotide biosynthesis. The ammonia molecule required for the HisF reaction is produced by the glutaminase subunit HisH (catalytic triad residues are depicted as spheres) and subsequently channeled to the active site of HisF (catalytic aspartate residues are depicted as spheres). In the absence of HisH, HisF can also use external ammonia that is added as ammonia salts. HisF adopts the $(\beta \alpha)_{8}$-barrel fold, an ubiquitous and catalytically versatile protein architecture ${ }^{21}$, which is considered one of the three most ancient protein folds ${ }^{22}$. HisH adopts the $\alpha / \beta$ hydrolase fold ${ }^{23}$.

superkingdoms is still under debate ${ }^{24}$, LUCA-HisF is among the oldest so far reconstructed proteins, if not the oldest hitherto calculated predecessor ${ }^{16,25,26}$. Among the 87 descendants of LUCA-HisF used for reconstruction, 78 of the 250 residues are less than $50 \%$ conserved, whereas 49 residues are strongly conserved. Accordingly, LUCA-HisF differs in 55 amino acids (22\%) from the closest BLAST match ${ }^{27}$, which is HisF from Thermovibrio ammonificans. The nucleotide and amino acid sequences of LUCA-HisF are given in the Supporting Information.

We have now produced LUCA-HisF in Escherichia coli, and analyzed its crystal structure, conformational stability, folding mechanism, and catalytic activity. The observed molecular characteristics of LUCA-HisF turned out to be similar to contemporary HisF proteins. Moreover, LUCA-HisF activates an extant HisH protein and thus comprises all elements required for allosteric interaction. Finally, we have also reconstructed and produced a LUCA-HisH protein and could show that it binds to LUCA-HisF with high affinity. Taken together, our results suggest that the protein inventory of the LUCA already contained elaborate enzyme complexes.

\section{Methods}

Cloning, expression, and purification of LUCA-HisF. The gene coding for LUCA-HisF was optimized for its expression in E. coli, synthesized (GeneArt), and cloned into the vector pET24a(+)(Stratagene) using the terminal restriction sites for NdeI and XhoI. Since the addition of a C-terminal hexahistidine tag to LUCA-HisF might influence its interaction with HisH proteins, a stop codon was integrated at the end of the gene. The gene was expressed in E. coli $\mathrm{T}_{7}$-Express cells (New England Biolabs) transformed with pET24a(+)LUCA-hisF. To this end, four liters of Luria Broth (LB) medium supplemented with $75 \mu \mathrm{g} / \mathrm{ml}$ kanamycin were inoculated with a pre-culture and incubated at $37{ }^{\circ} \mathrm{C}$. After an $\mathrm{OD}_{6 \text { oo }}$ of o.6 was reached, the temperature was lowered to $30^{\circ} \mathrm{C}$. Expression was induced by adding $0.5 \mathrm{mM}$ IPTG, and growth was continued overnight. Cells were harvested by centrifugation (Sorvall/RC5 $\mathrm{B}, \mathrm{GS}_{3}, 15 \mathrm{~min}, 4000 \mathrm{rpm}, 4{ }^{\circ} \mathrm{C}$ ), washed with $50 \mathrm{mM}$ potassium phosphate, $\mathrm{pH} 7.5$, and centrifuged again. The cells were suspended in the same buffer, lysed by sonification (Branson Sonifier W-25oD, 2 x 2 min in $15 \mathrm{sec}$ intervals, $45 \%$ pulse, $\mathrm{o}^{\circ} \mathrm{C}$ ), and centrifuged again (Sorvall/RC $5 \mathrm{~B}, \mathrm{SS}_{34}, 30 \mathrm{~min}, 13.000 \mathrm{rpm}, 4^{\circ} \mathrm{C}$ ) to separate the soluble from the insoluble fraction of the cell extract. In a first step, the soluble supernatant was subjected to ion exchange chromatography using a MonoQ column (HR 16/10, $20 \mathrm{ml}$, Pharmacia), which had been equilibrated with $50 \mathrm{mM}$ potassium phosphate, $\mathrm{pH}$ 7.5. The column was washed with equilibration buffer, and bound LUCA-HisF was eluted by applying a linear gradient of $\mathrm{O}-1.5 \mathrm{M} \mathrm{NaCl}$. Proteincontaining fractions were pooled, dialyzed against $50 \mathrm{mM}$ potassium phosphate, $\mathrm{pH} 7.5$, and subjected to ammonia sulfate precipitation. After $80 \%$ saturation with ammonia sulfate, precipitated protein was centrifuged (Sorvall/RC $5 \mathrm{~B}$, $\mathrm{SS}_{34}$, $30 \mathrm{~min}, 13.000 \mathrm{rpm}, 4{ }^{\circ} \mathrm{C}$ ), dissolved in $50 \mathrm{mM}$ potassium phosphate, $\mathrm{pH} 7.5,300 \mathrm{mM}$ potassium chloride, and finally purified via size exclusion chromatography. For this purpose a Superdex2oo column (HiLoad 26/6o, $320 \mathrm{ml}$, GE Healthcare) was operated with $50 \mathrm{mM}$ potassium phosphate, $\mathrm{pH} 7.5,300 \mathrm{mM}$ potassium chloride at $4{ }^{\circ} \mathrm{C}$. Fractions with pure protein were pooled and dialyzed against $50 \mathrm{mM}$ Tris/HCl, pH 7.5. According to SDS-PAGE (12.5\% acrylamide), LUCA-HisF was more than $95 \%$ pure. About $30 \mathrm{mg}$ of protein were obtained per liter of culture.

In order to determine the binding properties of LUCA-HisF to $\mathrm{HisH}$ proteins via fluorescence titration, all tryptophan residues of LUCA-HisF were replaced by tyrosines. Hence, LUCA_hisF_W138Y+W156Y was generated via overlap extension PCR ${ }^{2 \overline{8}}$ using pET24a(+)-LUCA-hisF as a template (see Supporting Information for oligonucleotide sequences), and subsequently cloned into $\mathrm{pET} 24 \mathrm{a}(+)$ via the terminal restriction sites for NdeI and XhoI. Expression and purification were performed as described for LUCA-HisF, yielding a comparable amount and purity of LUCA-HisF_W138Y+W156Y.

Cloning, expression, and purification of $z m \mathrm{HisH}$. Genomic DNA of Zymomonas mobilis (DSM424) was ordered from the Leibniz Institute DSMZ. In order to remove the internal restriction site for NdeI, the zmhisH gene was amplified by overlap extension PCR ${ }^{28}$ (see Supporting Information for oligonucleotide sequences) and cloned into pET24a(+) using the terminal restriction sites for NdeI and XhoI. After transformation of $E$. coli strain BL21(DE3) (Stratagene), expression was carried out at $30{ }^{\circ} \mathrm{C}$ overnight in four liters of LB medium, supplemented with $75 \mu \mathrm{g} / \mathrm{ml}$ kanamycin. Protein purification was performed as described for LUCA-HisF including 
ion exchange chromatography using $50 \mathrm{mM}$ Tris/ $\mathrm{HCl}, \mathrm{pH}$ 9, as buffer, ammonia sulfate precipitation, size exclusion chromatography, and final dialysis against $50 \mathrm{mM}$ Tris $/ \mathrm{HCl}$, $\mathrm{pH}$ 7.5. According to SDS-PAGE (12.5\% acrylamide), $\mathrm{zmHisH}$ was more than $95 \%$ pure. About $8 \mathrm{mg}$ of protein were obtained per liter of culture.

\section{Sequence reconstruction, cloning, expression and purifi-} cation of LUCA-HisH. As for LUCA-HisF, the reconstruction of LUCA-HisH was based on the tree $t_{\text {His } F_{-H i s H}}$ (Figure $\mathrm{S} 1$ ), which is close to an accepted organism phylogeny. In comparison to the multiple sequence alignment (MSA) of extant HisF sequences, the 87 extant HisH sequences exhibit a significantly higher variability. In fact, 140 of 226 residues are less than $50 \%$ conserved. Furthermore, the MSA $\left(\mathrm{HisH}_{\text {ext }}\right)$ contains several gaps. Recently, it has been shown that a novel algorithm for the phylogeny-aware gap placement named PRANK ${ }^{29}$ improves MSA quality. This is why we used PRANK with the option -showanc to deduce LUCA-HisH from the MSA HisH $H_{\text {ext }}$ under the control of $t_{H_{i s F} F_{-} H_{i s H}}$ (Figure S2). The nucleotide and amino acid sequences of LUCA-HisH are given in the Supporting Information. The protein shares 123 of 226 residues (54\%) with the closest BLAST ${ }^{27}$ match, which is HisH from Syntrophothermus lipocalidus.

The gene coding for LUCA-HisH was optimized for its expression in E. coli, synthesized (GeneArt), and cloned into the vector pET24a $(+)$ (Stratagene) using the terminal restriction sites for NdeI and Xhol. (The gene encodes a Cterminal hexa-histidin tag; see Supporting Information). Subsequently, pET24a(+)-LUCA-hisH was used to transform E. coli strain BL21-Gold ( $\left.\mathrm{DE}_{3}\right)$ (Stratagene). Protein expression, harvesting of cells, and cell lysis were performed as described for LUCA-HisF. As LUCA-HisH showed a high thermal stability, most of the host proteins could be removed by heat denaturation $\left(70^{\circ} \mathrm{C}, 15 \mathrm{~min}\right)$ followed by centrifugation (Sorvall/RC 5 B, SS34, $30 \mathrm{~min}, 13.000 \mathrm{rpm}, 4{ }^{\circ} \mathrm{C}$ ). For further purification, the supernatant of the heat step was loaded onto a HisTrapFF crude column ( $5 \mathrm{ml}$; GE Healthcare), which had been equilibrated with $50 \mathrm{mM}$ potassium phosphate, $\mathrm{pH} 7.5,300 \mathrm{mM}$ potassium chloride and $10 \mathrm{mM}$ imidazole. After washing with equilibration buffer, the bound protein was eluted by applying a linear gradient of 10$375 \mathrm{mM}$ imidazole. Fractions with pure protein were pooled, and LUCA-HisH was dialyzed against $10 \mathrm{mM}$ potassium phosphate, $\mathrm{pH}$ 7.5. As judged by SDS-PAGE, the protein was more than $95 \%$ pure. About 26 mg LUCA-HisH were obtained per liter of culture.

Crystallization, data collection, and refinement of LUCAHisF. Crystallization trials were carried out using the PEG/Ion screen (Hampton Research). The hanging drop vapor diffusion method was performed in 96-well plates (Greiner) at $291 \mathrm{~K}$. Drops contained $300 \mathrm{nl}$ of the respective reservoir buffer mixed with $300 \mathrm{nl}$ of LUCA-HisF $(13.9 \mathrm{mg} / \mathrm{ml})$ in $10 \mathrm{mM}$ potassium phosphate, $\mathrm{pH}$ 7.5. In each well equilibration was performed against $100 \mu \mathrm{l}$ of reservoir buffer. Crystals were obtained with $0.2 \mathrm{M}$ sodium phosphate monobasic monohydrate, $\mathrm{pH} 4.7$ and $20 \% \mathrm{wt} / \mathrm{vol} \mathrm{PEG} 3350$. After flash freezing in liquid nitrogen, data of single crystals were collected at the synchrotron beamlinePX2 (SLS) at $100 \mathrm{~K}$. Data were processed using XDS ${ }^{30}$ and the data quality assessment was done using phenix.xtriage ${ }^{31}$. Molecular re- placement was performed with MOLREP within the CCP4i suite ${ }^{32}$. A homology model of LUCA-HisF with HisF from Thermotoga maritima (tmHisF) (PDB ID 1THF) was built with MODELLER ${ }^{33}$ and served as a search model. Initial refinement was performed using REFMAC ${ }^{34}$. The model was further improved in several refinement rounds using automated restrained refinement with the program PHENIX ${ }^{31}$ and interactive modeling with Coot ${ }^{35}$. The data collection and refinement statistics are summarized in Table S1. The final model was analyzed using the program MolProbity ${ }^{36}$.

Analysis of the thermal stability of LUCA-HisF and LUCAHisH. Differential scanning calorimetry (DSC) was performed with LUCA-HisF in $50 \mathrm{mM}$ potassium phosphate, $\mathrm{pH} 7.5$, by heating the sample in a CSC 5100 Nano differential scanning calorimeter with a scan rate of $1{ }^{\circ} \mathrm{C} \mathrm{min}^{-1}$. The DSC data were analyzed with the program CpCalc (version 2.1; Calorimetry Sciences Corporation, 1995) to determine apparent melting temperatures $\left(\mathrm{T}_{\mathrm{M}}^{\mathrm{app}} \mathrm{DSC}\right)$. Thermal denaturation traces of LUCA-HisF and LUCA-HisH in $50 \mathrm{mM}$ potassium phosphate, $\mathrm{pH} 7.5$, were monitored with a JASCO J-815 circular dichroism (CD) spectrometer in a $0.1 \mathrm{~cm}$ cuvette by following the loss of ellipticity at $220 \mathrm{~nm}$. Unfolding was induced by raising the temperature in $1{ }^{\circ} \mathrm{C}$ increments at a ramp rate of $1{ }^{\circ} \mathrm{C}$ min ${ }^{1}$ with a Peltier-effect temperature controller. The midpoint temperatures of the unfolding transitions $\left(\mathrm{T}_{\mathrm{M}}^{\text {app }} \mathrm{CD}\right)$ were determined. Data are shown in Figure $\mathrm{S}_{3}$. The irreversibility of the denaturation traces precluded the thermodynamic analyses of the DSC and CD unfolding measurements.

Equilibrium unfolding/refolding transitions and formation of a burst-phase intermediate by LUCA-HisF. The thermodynamic stability of LUCA-HisF was determined by $\mathrm{GdmCl}-$ induced equilibrium unfolding transitions. The loss of tertiary structure was probed by protein fluorescence, the loss of secondary structure by far-UV CD. Samples with $2 \mu \mathrm{M}$ protein were prepared in $50 \mathrm{mM}$ Tris/ $\mathrm{HCl}$ buffer $(\mathrm{pH} 7.5)$ containing different concentrations of $\mathrm{GdmCl}$. $\mathrm{GdmCl}$ (ultrapure) was purchased from MP Biomedicals (Illkrich, France) and its concentration was determined by the refractive index of the solution ${ }^{37}$. To reach equilibrium, LUCAHisF was preincubated at the indicated concentration of $\mathrm{GdmCl}$ for $24 \mathrm{~h}$ at $25^{\circ} \mathrm{C}$.

The fluorescence emission signal at $320 \mathrm{~nm}$ (bandwidth 5 $\mathrm{nm}$ ) after excitation at $280 \mathrm{~nm}$ (bandwidth $3 \mathrm{~nm}$ ) was monitored with a JASCO model FP-650o spectrofluorimeter. The equilibrium unfolding transition of LUCA-HisF obtained by monitoring fluorescence is shown in Figure $\mathrm{S}_{4} \mathrm{~A}$ in comparison to $t m H i s F$ and its artificially designed precursors Symı and Sym2, which were constructed by duplication and fusion of the C-terminal half-barrel HisF-C followed by the optimization of the initial construct ${ }^{38-40}$. The transitions were analyzed according to the two-state equilibrium model, assuming a linear dependency of the free-energy of unfolding on the $\mathrm{GdmCl}$ concentration ${ }^{41}$. The obtained values for $\Delta \mathrm{G}_{\mathrm{D}}, \mathrm{m}$, and $[D]_{1} / 2$ are listed in Table $S_{2}$.

The far-UV circular dichroism (CD) signal at $225 \mathrm{~nm}$ was monitored using a JASCO model J815 CD spectrophotometer (path-length $5 \mathrm{~mm}$; bandwidth $1 \mathrm{~nm}$ ). The equilibrium unfolding/refolding transitions of LUCA-HisF obtained by 

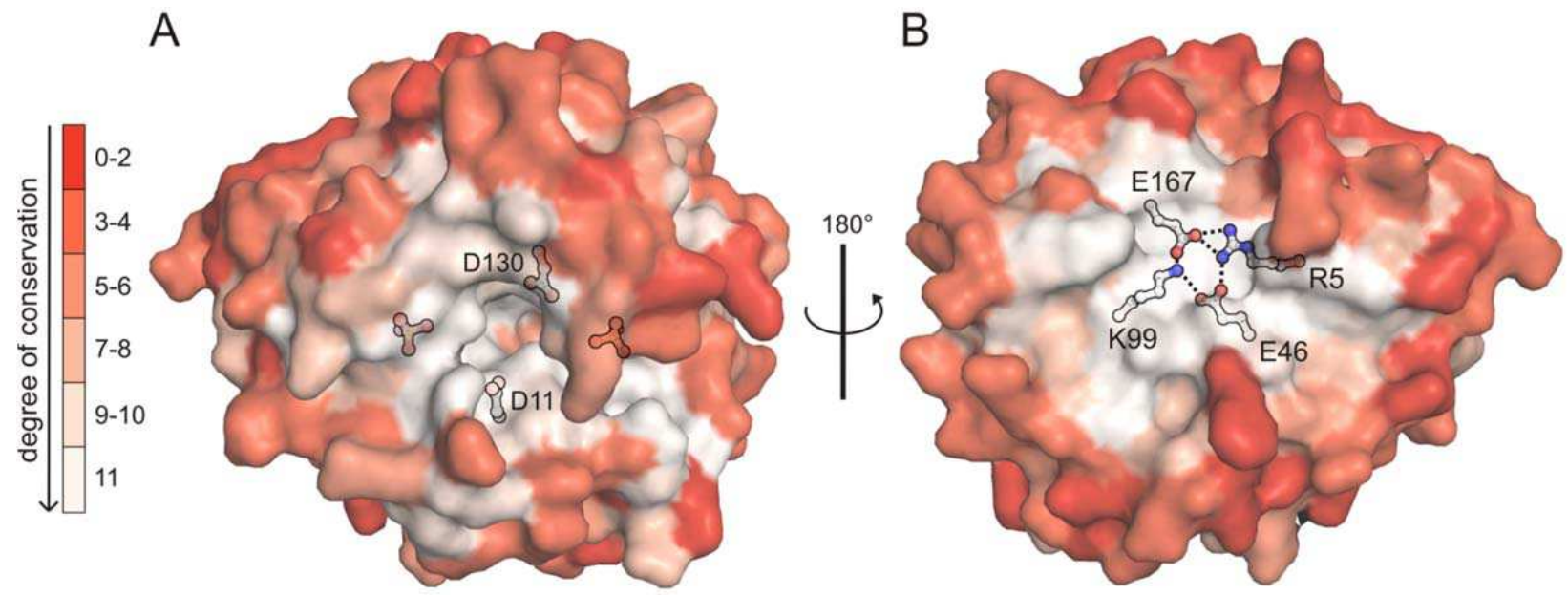

Figure 2. Crystal structure of LUCA-HisF. The surface is color-coded according to residue conservation deduced from the MSA used for reconstruction. Conservation values [0-11] were determined by means of Jalview ${ }^{42}$; strictly conserved residues are white. (A) Catalytic face of HisF and view along the ammonia channel. The catalytically important aspartate residues Di1 and Dizo as well as two bound phosphate ions, which mimic the phosphate moieties of the substrate PRFAR, are shown as sticks. (B) Stability face and ammonia tunnel gate at the bottom of the $\beta$-barrel. The salt bridge cluster between the residues R5, E46, K99, and E167 (depicted as sticks; electrostatic interactions indicated by dashed lines) defines the entrance to the ammonia channel ${ }^{43}$.

monitoring the far-UV CD signal are shown in Figure $\mathrm{S}_{4} \mathrm{~B}$ as closed and open circles, respectively.

Kinetics of refolding of LUCA-HisF in Figure $\mathrm{S}_{4} \mathrm{~B}$ were obtained by following the far-UV CD signal for 200 seconds in manual mixing experiments at various concentrations of $\mathrm{GdmCl}$ and extrapolating the exponential curve to zero time. The observed amplitude was plotted as function of the $\mathrm{GdmCl}$ concentration and is shown in Figure $\mathrm{S}_{4} \mathrm{~B}$ as closed triangles. It was significantly lower than the amplitude observed in the refolding equilibrium transitions, indicating that the major part of the $\mathrm{CD}$ change occurred within the dead time of the manual mixing experiment. This is interpreted with the formation of a compact burst-phase refolding intermediate with a high content of secondary structure.

Fluorescence titration of $z m H i s H$ and LUCA-HisH with LUCA-HisF. Fluorescence titration was used to determine the binding stoichiometry and affinity of the LUCAHisF/zmHisH and LUCA-HisF/LUCA-HisH complexes, as in the course of complex formation a tryptophan residue lying at the HisH-interface is shielded from the solvent ${ }^{5}$. Hence, when titrating either $7 \mu \mathrm{M} z m$ HisH or $5 \mu \mathrm{M}$ LUCA-HisH in $50 \mathrm{mM}$ potassium phosphate, $\mathrm{pH} 7.5$ with LUCAHisF_W138Y+W156Y, the emission maxima shifted from $345 \mathrm{~nm}$ to $325 \mathrm{~nm}$ and from $345 \mathrm{~nm}$ to $329 \mathrm{~nm}$, respectively (excitation at $295 \mathrm{~nm}$ ). The decreases in fluorescence emission at $318 \mathrm{~nm}$ were plotted against the added amounts of LUCA-HisF_W138Y+W156Y and the resulting curves were analyzed with a quadratic fit. Both titrations were performed in triplicate.

Analysis of enzymatic activity in vitro, and in vivo. In vitro enzymatic activities were determined by steady-state kinetics. The ammonia- and glutamine-dependent conversions of PRFAR into ImGP and AICAR (HisF reaction) were measured spectrophotometrically at $300 \mathrm{~nm}$ as previously described ${ }^{5}$.
At $25{ }^{\circ} \mathrm{C}$, entire progress curves at four different PRFARconcentrations were recorded either in $50 \mathrm{mM}$ Tris-acetate, $\mathrm{pH} 8.5$ in presence of $100 \mathrm{mM}$ ammonium acetate (ammoniadependent cyclase reaction) or in $50 \mathrm{mM}$ Tris-acetate, $\mathrm{pH} 8.0$ in presence of $15 \mathrm{mM}$ glutamine and $2 \mu \mathrm{M} \mathrm{zmHisH}$ (glutamine-dependent cyclase reaction). In both cases an excess of HisA from T. maritima was added in order to synthesize PRFAR in situ from ProFAR ${ }^{44}$, and $0.5 \mu \mathrm{M}$ of LUCA-HisF were used to initiate the measurements. Data were analyzed with the integrated form of the Michaelis-Menten equation using the program $\operatorname{COSY}^{45}$ to obtain $\mathrm{k}_{\text {cat }}$ and $\mathrm{K}_{\mathrm{M}}{ }^{\text {PRFAR }}$. In case of the LUCA-HisF/LUCA-HisH complex $(10 \mu \mathrm{M})$, no glutamine-dependent cyclase activity could be determined in presence of $10 \mathrm{mM}$ glutamine and $100 \mu \mathrm{M}$ ProFAR. The glutaminase activity of $z m H i s H(1 \mu \mathrm{M})$ in complex with liganded LUCA-HisF $(2 \mu \mathrm{M} ; 40 \mu \mathrm{M}$ ProFAR) was measured in a coupled enzymatic assay as previously described ${ }^{5}$. At $25^{\circ} \mathrm{C}$, produced glutamate was oxidized by a molar excess of glutamate dehydrogenase (Roche) in $50 \mathrm{mM}$ Tricine hydroxide, $\mathrm{pH}$ 8.o. Thus, the reduction of the coenzyme NAD ${ }^{+}$to NADH could be monitored spectrophotometrically at $340 \mathrm{~nm}$. Three glutamine saturation curves were recorded and fitted with the Michaelis-Menten equation to obtain $\mathrm{k}_{\text {cat }}$ and $\mathrm{K}_{\mathrm{M}}{ }^{\mathrm{Gln}}$. In an identical setup, no glutaminase activity (12 mM glutamine) could be detected for LUCA-HisH $(20 \mu \mathrm{M})$ in complex with ligand-bound LUCA-HisF ( $20 \mu \mathrm{M} ; 200 \mu \mathrm{M}$ ProFAR). The extent to which LUCA-HisF liganded with ProFAR activates $\mathrm{zmHisH}$ had to be determined in a discontinuous assay, since $\mathrm{NAD}^{+}$also exhibits a stimulating effect on glutaminase activity ${ }^{6}$. To this end, $10 \mathrm{mM}$ glutamine were incubated at $25^{\circ} \mathrm{C}$ with $0.5 \mu \mathrm{M} z m H i s H$ and $5 \mu \mathrm{M}$ LUCA-HisF either in the absence of ProFAR or in the presence of $40 \mu \mathrm{M}$ ProFAR. $150 \mu \mathrm{l}$ aliquots of the reaction mixture were collected after 15 , 30, 45 and 6o (only in the absence of ProFAR) minutes and spun through a $10 \mathrm{kDa}$ filter (Roth) to remove the enzymes. 
Table 1. Steady-state kinetic constants of the ImGP synthase pairs LUCA-HisF/zmHisH and $t m H i s F / t m H i s H$

\begin{tabular}{|c|c|c|c|}
\hline Ammonia-dependent cyclase activity $^{\mathrm{a}}$ & $\mathrm{k}_{\mathrm{cat}}, \mathrm{s}^{-1}$ & $\mathrm{~K}_{\mathrm{M}}^{\text {PRFAR }}, \mu \mathrm{M}$ & $\mathrm{k}_{\mathrm{cat}} / \mathrm{K}_{\mathrm{M}}^{\text {PRFAR }}, \mathrm{M}^{-1} \mathrm{~s}^{-1}$ \\
\hline \multirow{2}{*}{$\begin{array}{l}\text { LUCA-HisF } \\
\text { tmHisF }^{\text {b }}\end{array}$} & $0.078( \pm 0.003)$ & $0.29( \pm 0.04)$ & $2.8( \pm 0.3) \times 10^{5}$ \\
\hline & 1.2 & 3.6 & $3.3 \times 10^{5}$ \\
\hline Glutamine-dependent cyclase activity $^{\mathrm{c}}$ & $\mathrm{k}_{\mathrm{cat}}, \mathrm{s}^{-1}$ & $\mathrm{~K}_{\mathrm{M}}^{\text {PRFAR }}, \mu \mathrm{M}$ & $\mathrm{k}_{\mathrm{cat}} / \mathrm{K}_{\mathrm{M}}^{\text {PRFAR }}, \mathrm{M}^{-1} \mathrm{~s}^{-1}$ \\
\hline \multirow{2}{*}{$\begin{array}{l}\text { LUCA-HisF/zmHisH } \\
\text { tmHisF/tmHisH }{ }^{\mathrm{b}}\end{array}$} & $0.058( \pm 0.006)$ & $0.36( \pm 0.07)$ & $1.6( \pm 0.3) \times 10^{5}$ \\
\hline & 1.1 & 2.0 & $5.5 \times 10^{5}$ \\
\hline Glutaminase activity $^{\mathrm{d}}$ & $\mathrm{k}_{\mathrm{cat}}, \mathrm{s}^{-1}$ & $\mathrm{~K}_{\mathrm{M}}^{\mathrm{Gln}}, \mathrm{mM}$ & $\mathrm{k}_{\text {cat }} / \mathrm{K}_{\mathrm{M}}^{\mathrm{Gln}}, \mathrm{M}^{-1} \mathrm{~s}^{-1}$ \\
\hline \multirow{2}{*}{$\begin{array}{l}\text { LUCA-HisF/zmHisH } \\
\text { tmHisF/tmHisH }{ }^{\mathrm{b}}\end{array}$} & $0.21( \pm 0.03)$ & $1.9( \pm 0.9)$ & $1.2( \pm 0.3) \times 10^{2}$ \\
\hline & 0.1 & 0.8 & $1.3 \times 10^{2}$ \\
\hline Stimulation of glutaminase activity ${ }^{\mathrm{d}}$ & $\begin{array}{l}\mathrm{k}_{\mathrm{cat}}, \mathrm{s}^{-1} \\
\text { (without ProFAR) }\end{array}$ & $\begin{array}{l}\mathrm{k}_{\text {cat }}, \mathrm{s}^{-1} \\
\text { (ProFAR saturated) }\end{array}$ & Stimulation factor ${ }^{\mathrm{e}}$ \\
\hline \multirow{2}{*}{$\begin{array}{l}\text { LUCA-HisF/zmHisH } \\
\text { tmHisF/tmHisH }{ }^{\mathrm{b}}\end{array}$} & $3.85( \pm 0.04) \times 10^{-2}$ & $0.483( \pm 0.006)$ & 13 \\
\hline & $3.3 \times 10^{-4}$ & 0.1 & 303 \\
\hline
\end{tabular}

a Reaction conditions: $50 \mathrm{mM}$ Tris-acetate buffer, $\mathrm{pH} 8.5$ at $25^{\circ} \mathrm{C}$

${ }^{\mathrm{b}}$ Data taken from ref. ${ }^{6}$.

${ }^{c}$ Reaction conditions: $50 \mathrm{mM}$ Tris-acetate buffer, $\mathrm{pH} 8.0$ at $25{ }^{\circ} \mathrm{C}$

${ }^{\mathrm{d}}$ Reaction conditions: $50 \mathrm{mM}$ Tricine hydroxide, $\mathrm{pH} 8.0$ at $25^{\circ} \mathrm{C}$

${ }^{\mathrm{e}}$ The stimulation factor is the quotient $\mathrm{k}_{\mathrm{cat}}$ (ProFAR saturated)/ $\mathrm{k}_{\text {cat }}$ (without ProFAR)

$\mathrm{V}_{\max }$ was calculated from the linear increase of glutamate production with time, which was determined with the help of $1 \mathrm{mg} / \mathrm{ml}$ glutamate dehydrogenase and $0.7 \mathrm{mM} \mathrm{APAD}^{+}$ (Sigma) (the reaction mixture was diluted 1:7.5, and absorption was measured at $363 \mathrm{~nm}$ ). All measurements were performed in triplicates. ProFAR to PRFAR isomerisation activity (HisA reaction) was measured with the enzymatic assay described for the ammonia-dependent HisF reaction, however in presence of an excess of HisF ${ }^{46}$. PRA to CdRP isomerisation activity (TrpF reaction) was followed at $25^{\circ} \mathrm{C}$ by a fluorimetric assay (excitation at $350 \mathrm{~nm}$, emission at $400 \mathrm{~nm}$ ) $47,4^{8}$. The substrate PRA was generated in situ by $1 \mu \mathrm{M}$ yeast anthranilate phosphoribosyl transferase from anthranilate and PRPP, which was provided in a 30 -fold molar excess. Moreover, 2.5 $\mu \mathrm{M}$ indole-3-glycerol phosphate synthase from T. maritima were added to prevent product inhibition.

To test for enzymatic activity in vivo, the gene coding for LUCA-HisF was subcloned into the pTNA vector, which allows for constitutive expression in $E$. coli ${ }^{49}$. The resulting pTNA-LUCA-hisF plasmid was used to transform cells of auxotrophic $\Delta$ hisF, $\Delta$ hisA or $\Delta \operatorname{trpF}$ E. coli strains ${ }^{50,51}$. These strains lack the his $\mathrm{F}$, hisA, or $\operatorname{trpF}$ gene on their chromosome and are, therefore, unable to grow on medium without histidine or tryptophan, respectively. Growth experiments and controls were performed as described ${ }^{50}$.

\section{Results and Discussion}

Structure determination of LUCA-HisF. The gene coding for LUCA-HisF was synthesized, cloned into a plasmid, and expressed in E. coli. The LUCA-HisF protein was predominantly found in the soluble fraction of the host cell extract, and purified in a three step process using ion exchange chromatography, ammonia sulfate precipitation, and size exclusion chromatography. Purified LUCA-HisF was crystallized and its three-dimensional structure was determined at $1.48 \AA$ resolution by molecular replacement based on the structure of $t m \mathrm{HisF}^{52}$ (Figure 2, Table S1). LUCA-HisF (PDB ID ${ }_{4}$ EVZ) adopts the conserved $(\beta \alpha)_{8}$-barrel structure observed in the three extant HisF proteins from Pyrobaculum aerophilum (PDB ID ${ }_{1} \mathrm{H}_{5} \mathrm{Y}$ ), Thermus thermophilus (PDB ID 1KA9), and T. maritima (PDB ID ITHF), for which crystal structures have been previously determined. The superposition of LUCA-HisF with each of these structures by means of STAMP ${ }^{53}$ resulted in an overall root-mean-square deviation (RMSD) ranging from $1.14 \AA$ to $1.43 \AA$. In agreement with the postulated evolution of the $(\beta \alpha)_{8}$-barrel fold from a $(\beta \alpha)_{4^{-}}$ half-barrel ${ }^{54,55}$, LUCA-HisF displays a clear two-fold symmetry: The superposition of its N-terminal $\left[(\beta \alpha)_{1-4}\right]$ and $C$ terminal $\left[(\beta \alpha)_{5-8}\right]$ halves yielded an RMSD of $1.68 \AA$, which is similar to the corresponding values for the three extant HisF proteins $\left(1.27 \AA\right.$ for $1 \mathrm{H}_{5} \mathrm{Y}, 1.52 \AA$ for $1 \mathrm{KA} 9$, and $1.69 \AA$ for 1THF). Consistent with the internal symmetry, the two catalytically important aspartate residues ${ }^{5}$ are found on opposite sides of the active site at the C-terminal ends of $\beta$-strand 1 and $\beta$-strand 5 . Likewise, the two co-crystallized phosphate groups, which represent the two phosphate groups of the substrate PRFAR (Figure 1), are anchored by the C-terminal ends of $\beta$-strands 3 and 4 , and $\beta$-strands 7 and 8 , respectively (Figure 2A). Moreover, a stabilizing salt-bridge cluster at the $\mathrm{N}$-terminal end of the $\beta$-barrel, which contains four charged and invariant residues, which form the gate to the cyclase ammonia channel ${ }^{6,38,43}$, is also present in LUCA-HisF (Figure 2B).

Stability and folding mechanism of LUCA-HisF. The thermal stability of LUCA-HisF was determined by differential scanning calorimetry (DSC), which monitors overall unfolding, and the heat-induced decrease of the far-UV CD signal, which indicates the loss of secondary structure. The combination of both methods showed that thermal unfolding of LUCA-HisF is a two-step process with apparent transition 


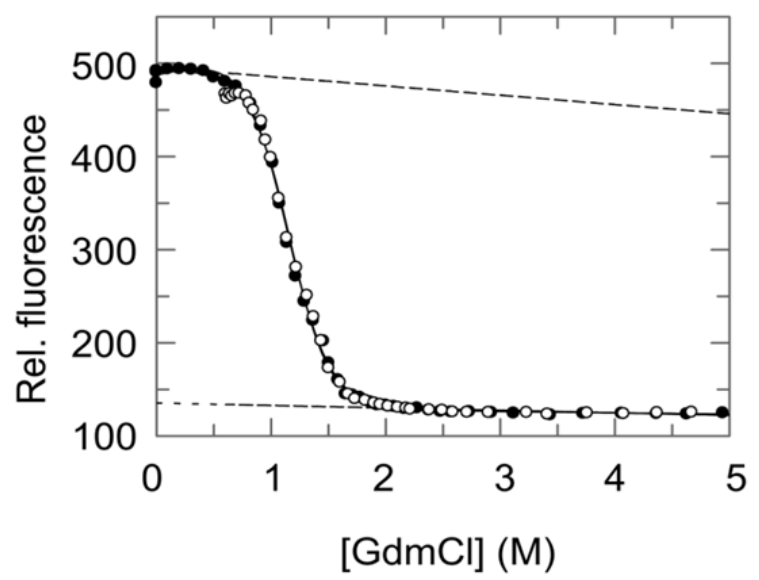

Figure 3. GdmCl-induced equilibrium unfolding/refolding transitions of LUCA-HisF. The transitions were followed by $\mathrm{Trp} / \mathrm{Tyr}$ fluorescence (excitation at $280 \mathrm{~nm}$; emission at $320 \mathrm{~nm}$ ) in $50 \mathrm{mM}$ Tris $/ \mathrm{HCl}$ buffer, $\mathrm{pH} 7 \cdot 5$. Closed symbols represent the unfolding experiment, started with folded protein, and open symbols represent the refolding experiment, started with protein that was previously unfolded in 6.o M GdmCl. The continuous line represents a fit to the unfolding transition on the basis of the two-state model. The dashed lines indicate the baselines for the pure $\mathrm{N}$ and $\mathrm{U}$ states. The thermodynamic parameters deduced from the analysis are given in the text and listed in Table S2.

midpoints of about $70{ }^{\circ} \mathrm{C}$ and $100{ }^{\circ} \mathrm{C}$ (Figure $\mathrm{S}_{3} \mathrm{~A}+\mathrm{B}$ ). These results characterize LUCA-HisF as an enzyme with a high resistance to heat. Interestingly, even higher denaturation temperatures were previously observed for enzymes from the common ancestors of Bacteria, Archaea and Archaea/Eukaryota ${ }^{16}$. These findings and our results are in agreement with rRNA and protein sequence analyses which have provided independent support for the increase of thermotolerance from the LUCA to the ancestors of Bacteria and Archaea-Eukaryota ${ }^{56}$. Furthermore, the conformational stability of LUCA-HisF was analyzed by $\mathrm{GdmCl}$-induced equilibrium unfolding and refolding transitions. The loss or gain of tertiary structure was probed by protein (Tyr/Trp) fluorescence. The equilibrium unfolding and refolding curves superpose well, which proves the reversibility of unfolding (Figure 3). Moreover, the transitions are adequately described by the two-state model ${ }^{41}$, indicating that no significant amounts of stable equilibrium intermediates are populated. The analysis yielded an m-value of $\sim 15 \mathrm{~kJ} \mathrm{~mol}^{-1} \mathrm{M}^{-1}$, a transition midpoint $\left([\mathrm{D}]_{1} / 2\right)$ at $1.2 \mathrm{M} \mathrm{GdmCl}$, and a free energy of unfolding in the absence of denaturant $\left(\Delta \mathrm{G}_{\mathrm{D}}\right)$ of $18 \mathrm{~kJ}$ $\mathrm{mol}^{-1}$. LUCA-HisF has a lower $\Delta \mathrm{G}_{\mathrm{D}}$ but a comparably high mvalue as $t m H i s F$ and its artificially designed precursors Symı and Sym2 ${ }^{39,40}$, indicating that it is comparably compact as these proteins but less stable (Figure $\mathrm{S}_{4} \mathrm{~A}$, Table S2). Folding and unfolding kinetics followed by Tyr/Trp fluorescence, showed that the reduced stability of LUCA-HisF is due to strongly increased unfolding rates of LUCA-HisF in comparison to tmHisF (Figure $\mathrm{S}_{5} \mathrm{~A}$ ). The comparison of the refolding kinetics of LUCA-HisF, tmHisF, Sym1, and Sym2 followed by fluorescence and far-UV CD (Supporting Information) showed that all four proteins share a common sequential folding mechanism including a non-productive burst-phase intermediate (Figure $\mathrm{S}_{4} \mathrm{~B}$ ) and two productive intermediates (Figure $\mathrm{S}_{5}$ ). The rate-limiting step that synchronizes folding is conserved (Figure $\mathrm{S} 6$ ).

Catalytic activity of LUCA-HisF. The enzymatic activity of LUCA-HisF was measured in vitro using steady-state kinetics. The analysis of PRFAR to ImGP/AICAR progress curves obtained in the presence of saturating concentrations of externally added ammonia (ammonia-dependent cyclase activity) yielded a catalytic efficiency $\left(\mathrm{k}_{\text {cat }} / \mathrm{K}_{\mathrm{M}}{ }^{\text {PRAR }}\right)$ of $2.8 \times 10^{5} \mathrm{M}^{-1} \mathrm{~s}^{-1}$, which is similar to the catalytic efficiency of $3.3 \times 10^{5} \mathrm{M}^{-1} \mathrm{~s}^{-1}$ that was obtained for HisF from tmHisF (Table 1). As ancient enzymes have been proposed to be less specific (more promiscuous) than their modern descendants ${ }^{57}$, we tested LUCA-HisF for its ability to catalyze related metabolic reactions. The homologous enzyme HisA, which precedes HisF in the histidine biosynthesis pathway, catalyzes the Amadori rearrangement of ProFAR to PRFAR. HisA shares with HisF the overall $(\beta \alpha)_{8}$-barrel fold as well as the location of the two symmetry-related catalytic aspartate residues and phosphate binding sites ${ }^{52}$. Phosphoribosyl anthranilate (PRA) isomerase (TrpF) catalyzes an Amadori rearrangement in tryptophan biosynthesis analogous to HisA in histidine biosynthesis ${ }^{49}$. Remarkably, a single amino acid exchange in the HisA and HisF proteins from T. maritima leads to TrpF activity, suggesting that these three phosphate-binding $(\beta \alpha)_{8}$-barrel proteins have evolved from a common precursor ${ }^{48,58}$. We examined LUCA-HisF for the isomerisation activity towards ProFAR and PRA. However, no substrate turnover could be detected, even in the presence of $50 \mu \mathrm{M}$ protein. These findings were complemented by assessing catalytic activity in vivo using metabolic selection. For this purpose, a plasmid harboring the LUCA-HisF gene was used to transform auxotrophic E. coli strains lacking either the intrinsic hisF, hisA, or $\operatorname{trpF}$ gene. When plated on minimal medium without histidine or tryptophan, the $\Delta$ hisF cells formed visible colonies within 24 hours, whereas the $\Delta$ hisA and $\Delta t r p F$ cells did not grow within one week. Taken together, these results demonstrate that LUCA-HisF is a monofunctional enzyme.

Formation of LUCA-HisF/HisH complexes. In order to test whether LUCA-HisF contains all structural elements required for complex formation, substrate channeling and allosteric communication, we assayed its functional interaction with the extant zmHisH enzyme from Zymomonas mobilis. For this purpose, zmHisH was produced in E. coli and purified. The binding of zmHisH to LUCA-HisF was analyzed via fluorescence titration ${ }^{5}$, which showed that the two proteins form a stoichiometric complex with a thermodynamic dissociation constant $\left(K_{\mathrm{D}}\right)$ of $113 \mathrm{nM}$ (Figure $\left.4 \mathrm{~A}\right)$.

The steady-state kinetic constants $k_{\text {cat }}$ and $\mathrm{K}_{\mathrm{M}}{ }^{\text {PRFAR }}$ of LUCAHisF in presence of $z m H i s H$ and saturating concentrations of glutamine (glutamine-dependent cyclase activity) compare well with the above reported ammonia-dependent cyclase activity (Table 1). This outcome confirms the functionality of the LUCA-HisF/zmHisH complex, as ammonia produced at the active site of HisH by means of glutamine hydrolysis is used as efficiently by LUCA-HisF as externally added ammonia. Moreover, this finding suggests that ammonia is transported from HisH to the active site of the synthase through a molecular channel formed by the central $\beta$-barrel of HisF- 

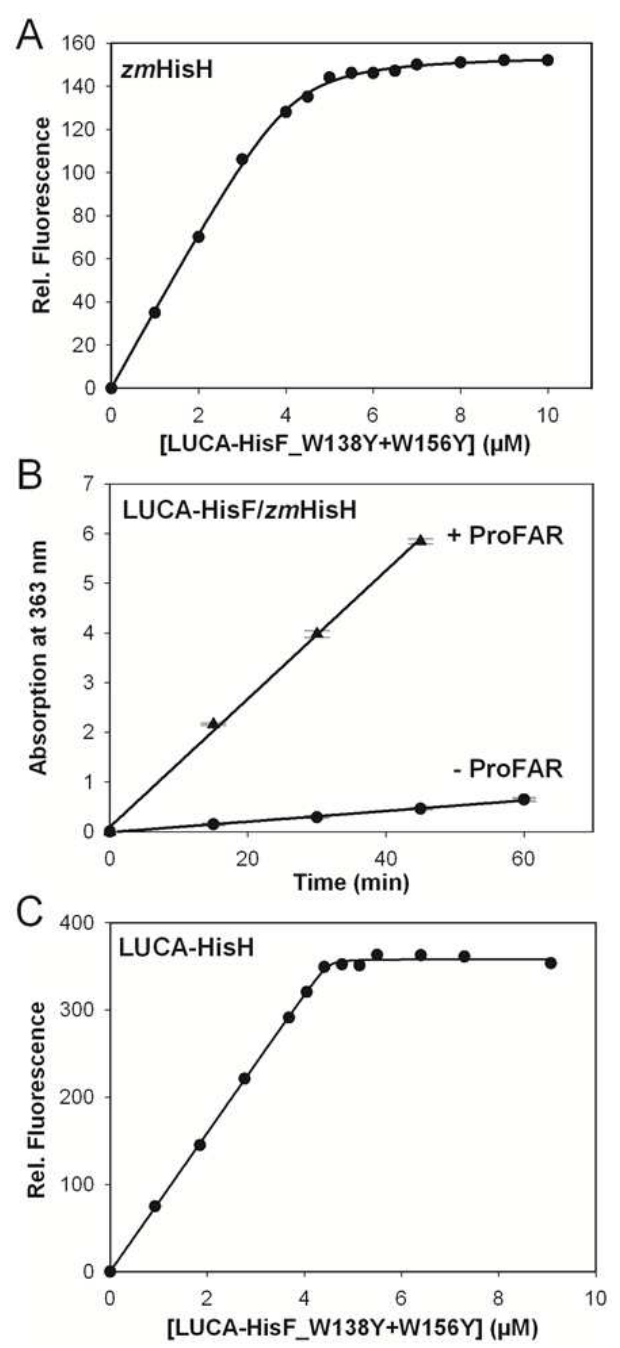

Figure 4. Fluorescence titration curve of zmHisH and LUCAHisH with LUCA-HisF and activation of $z m H i s H$ by LUCAHisF. (A) LUCA-HisF_W138Y+W156Y was added to $7 \mu \mathrm{M}$ $\mathrm{zmHisH}$ in $50 \mathrm{mM}$ potassium phosphate, $\mathrm{pH} 7.5,25^{\circ} \mathrm{C}$. Fluorescence emission at $318 \mathrm{~nm}$ was determined following excitation at $295 \mathrm{~nm}$. Quadratic fits of the obtained data points resulted in a $K_{D}$ value of $113( \pm 1) \mathrm{nM}$. The stoichiometry is slightly deviating from a 1:1 complex, indicating that a small fraction of $z m H i s H$ is not active. (B) Glutaminase activity of the LUCA-HisF/zmHisH complex in the absence (circles) and presence (triangles) of ProFAR was tested in a discontinuous assay (see Supplementary Materials for detailed information). Mean values and standard deviations of triplicate measurements are shown. $10 \mathrm{mM}$ glutamine were incubated with $0.5 \mu \mathrm{M} z m$ HisH and $5 \mu \mathrm{M}$ LUCA-HisF at $25^{\circ} \mathrm{C}$ in both cases. Glutaminase activity is enhanced 13 -fold in the presence of ProFAR (see Table 1). (C) Titration of $5 \mu \mathrm{M}$ LUCAHisH with LUCA-HisF_W138Y+W156Y was performed and analyzed analogous to (A), yielding a stoichiometric complex with a $K_{\mathrm{D}}$ value of $4( \pm 2) \mathrm{nM}$.

LUCA, as observed for extant HisF enzymes ${ }^{43,59}$. Furthermore, glutamine hydrolysis by $z m H i s H$ in presence of LUCAHisF and saturating concentrations of the substrate analogue ProFAR (glutaminase activity) is as efficient as glutaminase activity of HisH from T. maritima (tmHisH) in complex with ProFAR-liganded $t m H_{i s F}{ }^{4}$ (Table 1 ). The comparison of the $z m$ HisH activity in the presence and absence of ProFAR indicates a 13 -fold stimulation by the HisF-ligand in this nonnative complex (Figure ${ }_{4} \mathrm{~B}$ ), which is $\mathbf{2 3}$-fold lower than the stimulating effect of ProFAR in the native $t m \mathrm{HisF} / \mathrm{tmHisH}$ complex (Table 1).

Following the characterization of LUCA-HisF, we also reconstructed the amino acid sequence of the corresponding glutaminase LUCA-HisH. Again, we used the tree $t_{\text {HisF_HisH }_{-} \text {, but }}$ opted for the phylogeny-aware gap placement of PRANK ${ }^{29}$ to deduce LUCA-HisH from the MSA $\mathrm{HisH}_{\text {ext }}$, which contains several insertions and deletions. The gene coding for LUCAHisH was synthesized, cloned into a plasmid, and expressed in E. coli. The produced protein was soluble and could be purified by a combination of heat denaturation and $\mathrm{Ni}^{2+}$ affinity chromatography. As observed for LUCA-HisF, LUCAHisH exhibits a high thermotolerance. Unfolding followed by $\mathrm{CD}$ resulted in a single transition with a midpoint of about $79^{\circ} \mathrm{C}$ (Figure $\mathrm{S}_{3} \mathrm{C}$ ). Complex formation between LUCA-HisH and LUCA-HisF was probed by fluorescence titration ${ }^{5}$. Both proteins interacted stoichiometrically with very high affinity as demonstrated by a $\mathrm{K}_{\mathrm{D}}$ value of $4 \mathrm{nM}$ (Figure ${ }_{4} \mathrm{C}$ ). However, when testing the LUCA-HisF/LUCA-HisH complex for glutamine-dependent cyclase activity or LUCA-HisH for the hydrolysis of glutamine in the presence of LUCA-HisF and saturating concentrations of ProFAR, no enzymatic turnover could be determined. Thus, other than LUCA-HisF, LUCA$\mathrm{HisH}$ is catalytically inactive. As outlined in the following, uncertainties in the reconstruction process are probably responsible for this finding.

The evolutionary models underlying reconstruction consider each residue-position independently of all other positions. Thus, the reliability of a given reconstruction is not limited by sequence length, but by the composition of the MSA and the topology of the deduced phylogenetic tree. In the case of LUCA-HisF, 49 out of 250 residues are strictly conserved, among them are the two active site aspartate residues ${ }^{5}$ and amino acids contributing to the central ammonia channel ${ }^{38,43}$ (Figure 2). Furthermore, the four central nodes of $t_{\text {Hisf_HisH }}$ (Figure $\mathrm{S}_{1}$ ) possess posterior probabilities $\geq 0.88$. Taken together, these features suggest that tree topology and choice of the most likely residues for the corresponding predecessors and LUCA-HisF is largely unambiguous. In contrast, in the case of LUCA-HisH, only 21 out of 226 residues are strictly conserved, which makes the reconstruction much more prone to uncertainties.

Even more than a certain conservation of amino acid sequence composition, conservation of sequence length is an important prerequisite for a valid reconstruction. Along these lines, the sequence lengths of extant and reconstructed thioredoxins, which is the only other example for a fully functional enzyme from the LUCA era ${ }^{16}$, are very similar. In contrast, MSA His $\mathrm{H}_{\text {ext }}$ contains several gaps. Nonetheless, the phylogeny-aware gap placement by means of PRANK did allow us to reconstruct a stable LUCA-HisH protein with a fully functional protein-protein interface, albeit lacking enzyme activity. Obviously, $t_{\text {HisF_HisH }}$ was sufficiently informative to reconstruct ancestral residues at positions whose role did not change during evolution such as the catalytic triad ${ }^{3}$ and residues involved in binding of the substrate glutamine, 
as deduced from the structure of $t m \mathrm{HisH}^{6}$. In contrast, reconstruction seems to have failed at residue-positions that underwent frequent changes during evolution due to insertions and deletions. It has to be shown that highly articulated phylogenetic trees will enable us to reconstruct the correct series of indels and to further improve reconstruction for such difficult cases.

\section{Conclusions}

Taken together, LUCA-HisF, which presumably existed about 4 billion years ago, is similar to extant HisF proteins with respect to structure, stability, folding, and activity. Since similar results were obtained for predecessors of thioredoxin, ${ }^{16}$.experimental evidence accumulates for the existence of highly effective and non-promiscuous enzymes in the LUCA era. In addition, LUCA-HisF forms a stable complex with LUCA-HisH and a functional enzyme complex with the extant glutaminase $z m H i s H$. It is therefore plausible to assume that the evolution of the ImGP-S complex, including ammonia channeling and allosteric communication, has been completed in the LUCA era. Thus, our experimental findings are in line with the hypothesis that the LUCA had already a rather diverse metabolism, which was as sophisticated as are the metabolisms of its archaeal and bacterial successors ${ }^{60}$.

\section{ASSOCIATED CONTENT}

\section{Supporting Information}

Nucleotide and amino acid sequences of LUCA-HisF and LUCA-HisH. Oligonucleotides used for the construction of LUCA_hisF_W138Y+W156Y and for the genomic amplification of $\mathrm{zmHisH}$. Comparison of the folding mechanism of LUCA-HisF, T. maritima HisF, and its artificial precursors Symı and Sym2. Crystal structure determination of LUCAHisF (PDB ID 4EVZ): Data collection and refinement statistics (Table $\mathrm{S}_{1}$ ). Thermodynamic unfolding parameters of LUCA-HisF, T. maritima HisF, Sym1, and Sym2 (Table S2). Phylogenetic tree $\mathrm{t}_{\text {HisF_HisH }}$ used for the reconstruction of LUCA-HisF and LUCA-HisH (Figure S1). PRANK output for the reconstruction of LUCA-HisH (Figure S2). Thermal denaturation of LUCA-HisF and LUCA-HisH (Figure $\mathrm{S}_{3}$ ). Equilibrium unfolding transitions of LUCA-HisF, T. maritima HisF, Sym1, and Sym2, and formation of a burst-phase intermediate by LUCA-HisF (Figure $\mathrm{S}_{4}$ ). Apparent rate constants $(\lambda)$ and amplitudes of refolding and unfolding kinetics of LUCAHisF (Figure $\mathrm{S}_{5}$ ). Unifying folding mechanism for LUCAHisF, T. maritima HisF, and Symı and Sym2 (Figure S6). References for Supporting Information.This material is available free of charge via the Internet at http://pubs.acs.org.

\section{AUTHOR INFORMATION}

\section{Corresponding Authors}

Rainer.Merkl@ur.de and Reinhard.Sterner@ur.de

\section{Author Contributions}

The first two authors contributed equally. All authors have given approval to the final version of the manuscript.

\section{Notes}

The authors declare no competing financial interests.

\section{ACKNOWLEDGMENT}

B.R. and J.S. were supported by fellowships from the Cusanuswerk and the Fonds der Chemischen Industrie, respectively.

\section{REFERENCES}

(1) Zalkin, H. Adv. Enzymol. Relat. Areas Mol. Biol. 1993, 66, 203.

(2) Chittur, S. V.; Chen, Y.; Davisson, V. J. Prot. Expr. Purif. 2ooo, $18,366$.

(3) Myers, R. S.; Jensen, J. R.; Deras, I. L.; Smith, J. L.; Davisson, V. J. Biochemistry 2003, 42, 7013.

(4) Klem, T. J.; Davisson, V. J. Biochemistry 1993, 32, 5177.

(5) Beismann-Driemeyer, S.; Sterner, R. J. Biol. Chem. 20o1, 276, 20387 .

(6) List, F.; Vega, M. C.; Razeto, A.; Hager, M. C.; Sterner, R.;

Wilmanns, M. Chem. Biol. 2012, 19, 1589.

(7) Hanson-Smith, V.; Kolaczkowski, B.; Thornton, J. W. Mol. Biol. Evol. 2010, 27, 1988.

(8) Harms, M. J.; Thornton, J. W. Curr. Opin. Struct. Biol. 2o10, 2o, 360.

(9) Benner, S. A.; Sassi, S. O.; Gaucher, E. A. Adv. Enzymol. Relat. Areas Mol. Biol. 2007, 75, 1.

(10) Thomson, J. M.; Gaucher, E. A.; Burgan, M. F.; De Kee, D. W.; Li, T.; Aris, J. P.; Benner, S. A. Nat. Genet. 2oo5, 37, 630.

(11) Chang, B. S.; Jonsson, K.; Kazmi, M. A.; Donoghue, M. J.; Sakmar, T. P. Mol. Biol. Evol. 20o2, 19, 1483.

(12) Malcolm, B. A.; Wilson, K. P.; Matthews, B. W.; Kirsch, J. F.; Wilson, A. C. Nature 1990, 345, 86.

(13) Stackhouse, J.; Presnell, S. R.; McGeehan, G. M.; Nambiar, K. P.; Benner, S. A. FEBS Lett. 1990, 262, 104.

(14) Jermann, T. M.; Opitz, J. G.; Stackhouse, J.; Benner, S. A. Nature 1995, 374, 57.

(15) Finnigan, G. C.; Hanson-Smith, V.; Stevens, T. H.; Thornton, J. W. Nature 2012, 481, 360.

(16) Perez-Jimenez, R.; Inglés-Prieto, A.; Zhao, Z. M.; SanchezRomero, I.; Alegre-Cebollada, J.; Kosuri, P.; Garcia-Manyes, S.; Kappock, T. J.; Tanokura, M.; Holmgren, A.; Sanchez-Ruiz, J. M.; Gaucher, E. A.; Fernandez, J. M. Nat. Struct. Mol. Biol. 2011, 18, 592.

(17) Nisbet, E. G.; Sleep, N. H. Nature 20o1, 409, 1083.

(18) Richter, M.; Bosnali, M.; Carstensen, L.; Seitz, T.; Durchschlag, H.; Blanquart, S.; Merkl, R.; Sterner, R. J. Mol. Biol. 2010, 398, 763.

(19) Lartillot, N.; Philippe, H. Mol. Biol. Evol. 2004, 21, 1095.

(20) Blanquart, S.; Lartillot, N. Mol. Biol. Evol. 2oo8, 25, 842.

(21) Wierenga, R. K. FEBS Lett. 2001, 492, 193.

(22) Caetano-Anollés, G.; Kim, H. S.; Mittenthal, J. E. Proc. Natl. Acad. Sci. U S A $\mathbf{2 0 0 7}, 104,9358$.

(23) Ollis, D. L.; Cheah, E.; Cygler, M.; Dijkstra, B.; Frolow, F.; Franken, S. M.; Harel, M.; Remington, S. J.; Silman, I.; Schrag, J.; Sussman, J. L.; Verschueren, K. H. G.; Gioldman, A. Prot. Eng. 1992, $5,197$.

(24) Gribaldo, S.; Poole, A. M.; Daubin, V.; Forterre, P.; BrochierArmanet, C. Nat. Rev. Microbiol. 2o10, 8, 743.

(25) Gaucher, E. A.; Govindarajan, S.; Ganesh, O. K. Nature 2oo8, 451, 704 .

(26) Risso, V. A.; Gavira, J. A.; Mejia-Carmona, D. F.; Gaucher, E. A.; Sanchez-Ruiz, J. M. J. Am. Chem. Soc. 2013, 135, 2899.

(27) Boratyn, G. M.; Camacho, C.; Cooper, P. S.; Coulouris, G.; Fong, A.; Ma, N.; Madden, T. L.; Matten, W. T.; McGinnis, S. D.; Merezhuk, Y.; Raytselis, Y.; Sayers, E. W.; Tao, T.; Ye, J.; Zaretskaya, I. Nucleic Acids Res. 2013, 41, W29.

(28) Ho, S. N.; Hunt, H. D.; Horton, R. M.; Pullen, J. K.; Pease, L. R. Gene 1989, 77, 51.

(29) Löytynoja, A.; Goldman, N. Science 2oo8, 320, 1632.

(30) Kabsch, W. Acta Crystallogr. D Biol. Crystallogr. 2010, 66, 125. 
(31) Adams, P. D.; Grosse-Kunstleve, R. W.; Hung, L. W.; Ioerger, T. R.; McCoy, A. J.; Moriarty, N. W.; Read, R. J.; Sacchettini, J. C.; Sauter, N. K.; Terwilliger, T. C. Acta Crystallogr. D Biol. Crystallogr. 2002, 58, 1948.

(32) Potterton, L.; McNicholas, S.; Krissinel, E.; Gruber, J.; Cowtan, K.; Emsley, P.; Murshudov, G. N.; Cohen, S.; Perrakis, A.; Noble, M. Acta Crystallogr. D Biol. Crystallogr. 2004, 6o, 2288.

(33) Sali, A.; Blundell, T. L. J. Mol. Biol. 1993, 234, 779.

(34) Murshudov, G. N.; Vagin, A. A.; Dodson, E. J. Acta Crystallogr. D Biol. Crystallogr. 1997, 53, 240.

(35) Emsley, P.; Cowtan, K. Acta Crystallogr. D Biol. Crystallogr. 2004, 6o, 2126.

(36) Davis, I. W.; Leaver-Fay, A.; Chen, V. B.; Block, J. N.; Kapral, G. J.; Wang, X.; Murray, L. W.; Arendall, W. B., 3rd; Snoeyink, J.; Richardson, J. S.; Richardson, D. C. Nucleic Acids Res. 2007, 35, W 375 .

(37) Pace, C. N. Methods in Enzymology 1986, 131, 266.

(38) Höcker, B.; Lochner, A.; Seitz, T.; Claren, J.; Sterner, R. Biochemistry 2009, 48, 1145

(39) Carstensen, L.; Sperl, J. M.; Bocola, M.; List, F.; Schmid, F. X.; Sterner, R. J. Am. Chem. Soc. 2012, 134, 12786.

(40) Carstensen, L.; Zoldak, G.; Schmid, F. X.; Sterner, R. Biochemistry 2012, 51, 3420.

(41) Santoro, M. M.; Bolen, D. W. Biochemistry 1988, 27, 8063. (42) Waterhouse, A. M.; Procter, J. B.; Martin, D. M.; Clamp, M.; Barton, G. J. Bioinformatics 2009, 25, 1189.

(43) Douangamath, A.; Walker, M.; Beismann-Driemeyer, S.; VegaFernandez, M. C.; Sterner, R.; Wilmanns, M. Structure 2002, 10, 185.
(44) Thoma, R.; Obmolova, G.; Lang, D. A.; Schwander, M.; Jeno, P.; Sterner, R.; Wilmanns, M. FEBS Lett. 1999, 454, 1.

(45) Eberhard, M. Comput. Appl. Biosci. 1990, 6, 213.

(46) Jürgens, C.; Strom, A.; Wegener, D.; Hettwer, S.; Wilmanns, M.; Sterner, R. Proc. Natl. Acad. Sci. U S A 2000, 97, 9925.

(47) Hommel, U.; Eberhard, M.; Kirschner, K. Biochemistry 1995, 34, 5429 .

(48) Leopoldseder, S.; Claren, J.; Jürgens, C.; Sterner, R. J. Mol. Biol. 2004, 337, 871 .

(49) Henn-Sax, M.; Thoma, R.; Schmidt, S.; Hennig, M.; Kirschner, K.; Sterner, R. Biochemistry 2002, 41, 12032.

(50) Claren, J.; Malisi, C.; Höcker, B.; Sterner, R. Proc. Natl. Acad. Sci. U S A 2009, 106, 3704

(51) Sterner, R.; Dahm, A.; Darimont, B.; Ivens, A.; Liebl, W.; Kirschner, K. EMBO J. 1995, 14, 4395.

(52) Lang, D.; Thoma, R.; Henn-Sax, M.; Sterner, R.; Wilmanns, M. Science 2000, 289, 1546.

(53) Russell, R. B.; Barton, G. J. Proteins 1992, 14, 309.

(54) Höcker, B.; Beismann-Driemeyer, S.; Hettwer, S.; Lustig, A.; Sterner, R. Nat. Struct. Biol. 2oo1, 8, 32.

(55) Sterner, R.; Höcker, B. Chem. Rev. 2005, 105, 4038.

(56) Boussau, B.; Blanquart, S.; Necsulea, A.; Lartillot, N.; Gouy, M. Nature 2008, 456, 942.

(57) Jensen, R. A. Annu. Rev. Microbiol. 1976, 30, 409.

(58) List, F.; Sterner, R.; Wilmanns, M. ChemBioChem 2o11, $12,1487$.

(59) Chaudhuri, B. N.; Lange, S. C.; Myers, R. S.; Chittur, S. V.; Davisson, V. J.; Smith, J. L. Structure 20o1, 9, 987.

(6o) Glansdorff, N.; Xu, Y.; Labedan, B. Biol. Direct 2008, 3, 29.

Insert Table of Contents artwork here
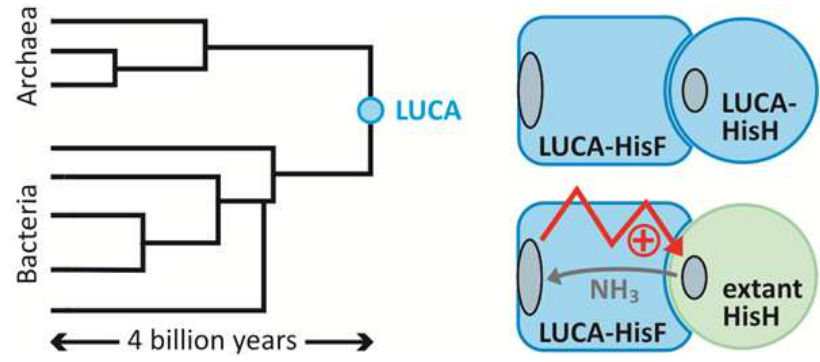